Proceedings of the

Combustion Institute

\title{
Turbulent flame speed and reaction layer thickening in premixed jet flames at constant Karlovitz and increasing Reynolds numbers
}

\author{
Antonio Attili ${ }^{\mathrm{a}, *}$, Stefano Luca ${ }^{\mathrm{b}}$, Dominik Denker ${ }^{\mathrm{a}}$, Fabrizio Bisetti ${ }^{\mathrm{c}}$, \\ Heinz Pitsch ${ }^{\text {a }}$ \\ a Institute for Combustion Technology, RWTH Aachen University, Aachen 52056, Germany \\ ${ }^{\mathrm{b}}$ King Abdullah University of Science and Technology (KAUST), Clean Combustion Research Center (CCRC), Thuwal \\ 23955, Saudi Arabia \\ ${ }^{\mathrm{c}}$ Department of Aerospace Engineering and Engineering Mechanics, University of Texas at Austin, Austin, TX 78712, USA
}

Received 7 November 2019; accepted 8 June 2020

\begin{abstract}
A series of Direct Numerical Simulations (DNS) of lean methane/air flames was conducted to investigate the enhancement of the turbulent flame speed and modifications to the reaction layer structure associated with the systematic increase of the integral scale of turbulence $l$ while the Karlovitz number and the Kolmogorov scale are kept constant. Four turbulent slot jet flames are simulated at increasing Reynolds number and up to $\operatorname{Re} \approx 22,000$, defined with the bulk velocity, slot width, and the reactants' properties. The turbulent flame speed $S_{T}$ is evaluated locally at selected streamwise locations and it is observed to increase both in the streamwise direction for each flame and across flames for increasing Reynolds number, in line with a corresponding increase of the turbulent integral scale. In particular, the turbulent flame speed $S_{T}$ increases exponentially with the integral scale for $l$ up to about 6 laminar flame thicknesses, while the scaling becomes a power-law for larger values of $l$. These trends cannot be ascribed completely to the increase in the flame surface, since the turbulent flame speed looses its proportionality to the flame area as the integral scale increases; in particular, it is found that the ratio of turbulent flame speed to area attains a power-law scaling ${ }^{0.2}$. This is caused by an overall broadening of the reaction layer for increasing integral scale, which is not associated with a corresponding decrease of the reaction rate, causing a net enhancement of the overall burning rate. This observation is significant since it suggests that a continuous increase in the size of the largest scales of
\end{abstract}

\footnotetext{
* Corresponding author.

E-mail address: a.attili@itv.rwth-aachen.de (A. Attili).
} 
turbulence might be responsible for progressively stronger modifications of the flame's inner layers even if the smallest scales, i.e., the Karlovitz number, are kept constant.

(C) 2020 The Author(s). Published by Elsevier Inc. on behalf of The Combustion Institute.

This is an open access article under the CC BY-NC-ND license.

(http://creativecommons.org/licenses/by-nc-nd/4.0/)

Keywords: Turbulent premixed flames; Direct numerical simulation; Turbulent flame speed; High Reynolds number; Flame thickening

\section{Introduction}

The propagation of premixed flames subject to the stirring and straining of a turbulent velocity field is known to be affected by two fundamental mechanisms [1-4]. Turbulence causes an increase of the area of the flame surface, contributing to an enhancement of the overall burning rate. In addition, turbulence can change the flame structure by perturbing the balance between reaction and diffusion, modifying the local burning rate, sometimes to the point of inducing local extinction, and variation of the diffusive fluxes, which are associated with perturbations to the local thickness of the different layers of the flame structure. A commonly accepted hypothesis is that the perturbation of the inner layer of the flame depends only on the Karlovitz number Ka, which parametrizes the ratio between the flame thickness $\delta_{L}$ and the size of the smallest turbulent scales $\eta$, while variations of Reynolds number Re and turbulence integral scale $l$ do not play a role if the Karlovitz number is kept constant [1].

In this work, we analyze the turbulent flame speed and the modifications of the inner reaction layer in a series of four jet flames characterized by nearly constant Karlovitz number and turbulent intensity (velocity root mean square) $u^{\prime}$, while the Reynolds number and turbulence integral scale vary significantly across the flame series. In particular, a ratio of integral scale, computed in the unburned gas, to laminar flame thermal thickness of $\approx 20$ was achieved at the largest jet Reynolds number of 22400. A previous analysis of the same Direct Numerical Simulation (DNS) dataset [5] focused on the growth rates of the area of the flame surface and demonstrated the scaling of flame stretch, and its components, with the Kolmogorov scale.

In addition to the relative scarcity of studies that isolated the effects of the integral scale on the turbulent flame speed and flame structure [5-8], this analysis is motivated by the recent effort of Skiba et al. [9] and Driscoll et al. [4] who demonstrated a transition from a flamelet regime towards a behavior characterized by thicker preheat layers for integral scales of increasing size at constant $u$, i.e., moving horizontally in the Borghi-Peters dia- gram of turbulent premixed combustion [1]. Starting from these observations, we investigate whether an increase in the size of the largest turbulent scale (a Reynolds number increase) promotes a modification to the structure of the flame, in terms of modification of the local burning rate and inner layer thickness, for a fixed size of the smallest scale relative to the laminar flame thickness (constant Karlovitz number). In addition, the spatial inhomogeneity and streamwise evolution of the turbulent flame speed and flame thickening is assessed.

\section{Configuration, numerical methods, and overview of the flames}

A slot turbulent premixed jet flame with equivalence ratio $\phi=0.7$, temperature $800 \mathrm{~K}$ and pressure of $4 \mathrm{~atm}$, surrounded by a coflow of burnt gases, is considered. A summary of all relevant flow parameters is provided in Table. 1. The database is described in details in Ref. [5]; therefore, only a breif summary is provided here. Based on onedimensional simulations of a freely propagating flame, the laminar flame speed is $S_{L}=1 \mathrm{~m} \mathrm{~s}^{-1}$ and the thermal thickness is $\delta_{L}=110 \mu \mathrm{m}$. The bulk velocity of the jet is $U=100 \mathrm{~m} \mathrm{~s}^{-1}$ and the coflow has a uniform velocity of $15 \mathrm{~m} \mathrm{~s}^{-1}$. For the four cases considered, the Reynolds number $\mathrm{Re}=$ $U H / v$ varies from 2800 to 22400 as the slot's width $H$ increases from 0.6 to $4.8 \mathrm{~mm}$ ( $v$ is the kinematic viscosity of the reactants).

The flame configurations were selected in order to vary the Reynolds number, while keeping the Karlovitz number $\mathrm{Ka}=\delta_{L}^{2} / \eta^{2}$ approximately constant. Because the turbulence intensity is a fraction of the bulk velocity with only a minor dependence on the Reynolds number, this scaling approach results in a nearly constant value of $u^{\prime} / S_{L}$ with increasing $l / \delta_{L}$ as the integral scale $l$ increases with the size of the jet width $H$. Fig. 1 shows the BorghiPeters regime diagram for the flames. Following Hawkes et al. [10], the lines at constant Karlovitz $\mathrm{Ka}$ and turbulent Reynolds number $\mathrm{Re}_{\mathrm{T}}=u^{\prime} l / v$ are plotted considering that $S_{L} \delta_{L} / v \approx 5.2$ for the unburned mixture and thermodynamics conditions of the present DNS and not unity as usually assumed to draw the diagram [1]. The diagram shows 
Table 1

Simulations parameters, evaluated at the crosswise location where $\langle c\rangle=c_{0}=0.73$ and streamwise location $x / l_{F}=0.6 . \eta$ is the Kolmogorov scale, $\operatorname{Re}_{\lambda}$ the Reynolds number based on the Taylor microscale, and $N_{x}, N_{y}, N_{z}$ the number of grid points in the three directions.

\begin{tabular}{lllll}
\hline & $\mathrm{R} 1$ & $\mathrm{R} 2$ & $\mathrm{R} 3$ & $\mathrm{R} 4$ \\
\hline $\mathrm{H}(\mathrm{mm})$ & 0.6 & 1.2 & 2.4 & 4.8 \\
$\mathrm{Re}$ & 2800 & 5600 & 11200 & 22400 \\
$u^{\prime}(\mathrm{m} / \mathrm{s})$ & 14.3 & 10.1 & 9.9 & 11.7 \\
$l(\mathrm{~mm})$ & 0.54 & 0.54 & 0.67 & 1.1 \\
$\eta(\mu \mathrm{m})$ & 18 & 23 & 25 & 25 \\
$u^{\prime} / S_{L}$ & 14.2 & 10.0 & 9.8 & 11.6 \\
$l / \delta_{L}$ & 4.8 & 4.8 & 5.9 & 9.5 \\
$\mathrm{Ka}$ & 39 & 23 & 21 & 21 \\
$\mathrm{Re}$ & 49 & 39 & 40 & 50 \\
$N_{x}$ & 720 & 1440 & 2880 & 5760 \\
$N_{y}$ & 480 & 960 & 1920 & 3840 \\
$N_{z}$ & 256 & 256 & 512 & 1024 \\
\hline
\end{tabular}

that the Karlovitz number is approximately constant, especially for the flames at high Reynolds number and large integral scale, while the integral scale and Reynolds number increase.

The size of the computational domain is $24 H$ in the streamwise (x), $16 H$ in the crosswise $(y)$, and

(a)

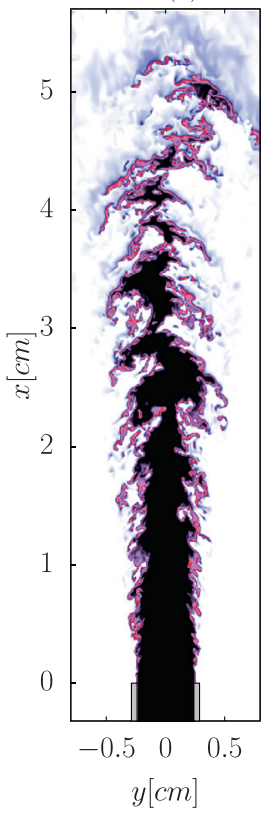

(b)

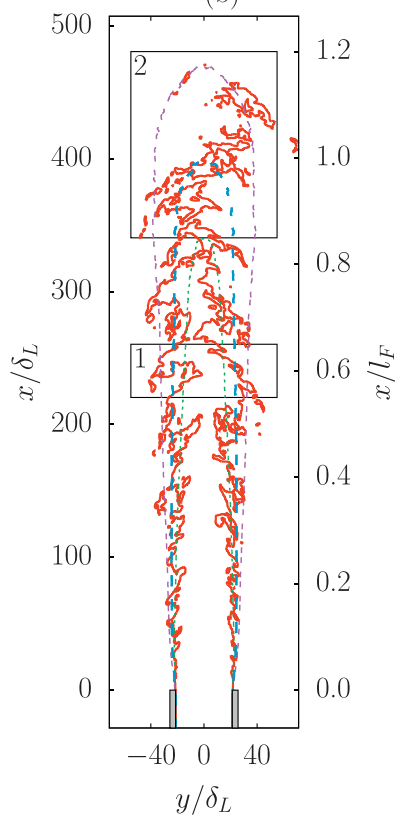

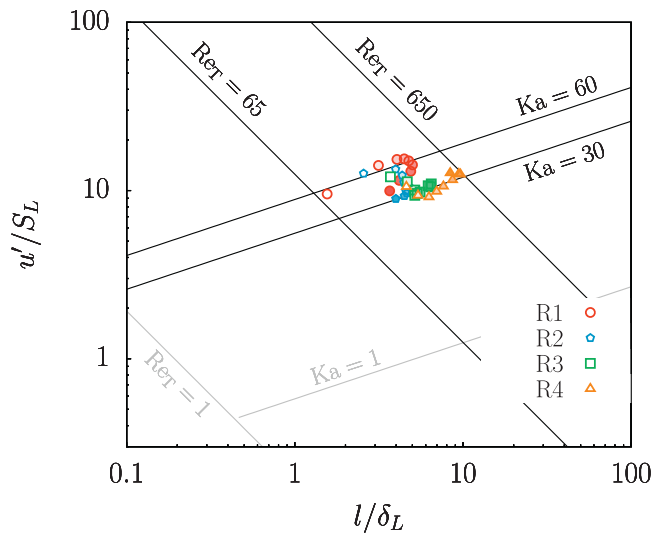

Fig. 1. Borghi-Peters diagram for the four flames. At each axial location, the value of the turbulent velocity fluctuations and integral scale are shown at the lateral location where $\langle c\rangle=0.73$. Light to dark color of the symbol filling indicates upstream to downstream positions.

$4.3 H$ in the spanwise $(z)$ direction $(8.5 H$ for $\mathrm{R} 1)$. The domain is periodic in $z$, open boundary conditions are prescribed at the outlet in $x$ and no-slip conditions are imposed at the boundaries in $y$. The

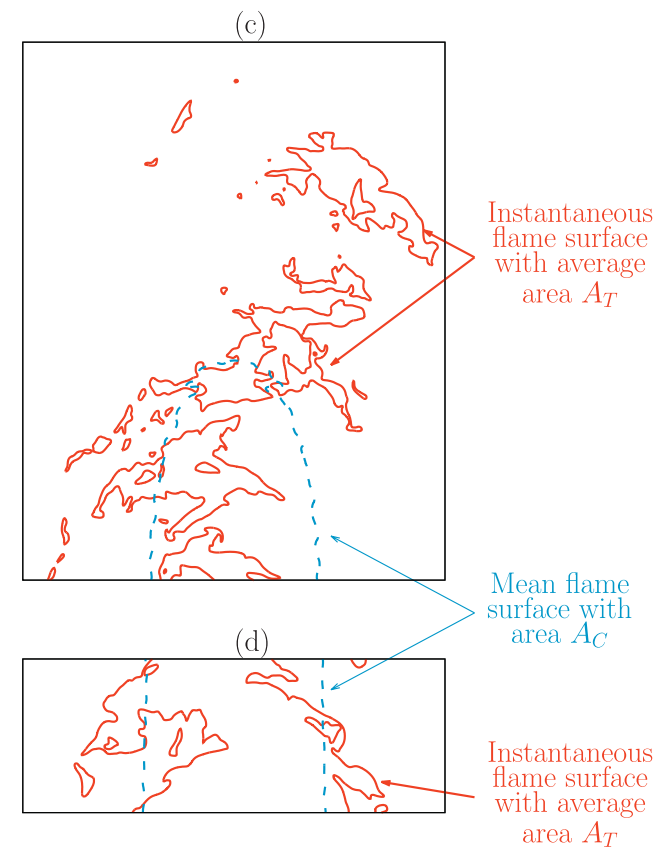

Fig. 2. (a) Visualization of the temperature field in a two-dimensional cut for the flame with the highest Reynnolds number (R4). The temperatures range from $800 \mathrm{~K}$ in the center of the jet to $2218 \mathrm{~K}$ in the coflow. (b) Isosurface of the instantaneous progress variable $c=0.73$ (red solid line) and three isosurface $(\langle c\rangle=0.5$ green dotted line, $\langle x\rangle=0.73$ blue dashed line, $\langle c\rangle=0.9$ purple dotted line) of the mean progress variable field. The axes are shown in physical units in (a) and normalized with the laminar flame thermal thickness $\delta_{L}$ in (b), where the streamwise direction $x$ is shown also normalized with the flame length $l_{F}$. (c,d) Enlargement of the volumes 1 and 2 in (b) showing the instantaneous and mean flame surface. Results are shown for the R4 flame. (For interpretation of the references to color in this figure legend, the reader is referred to the web version of this article.) 

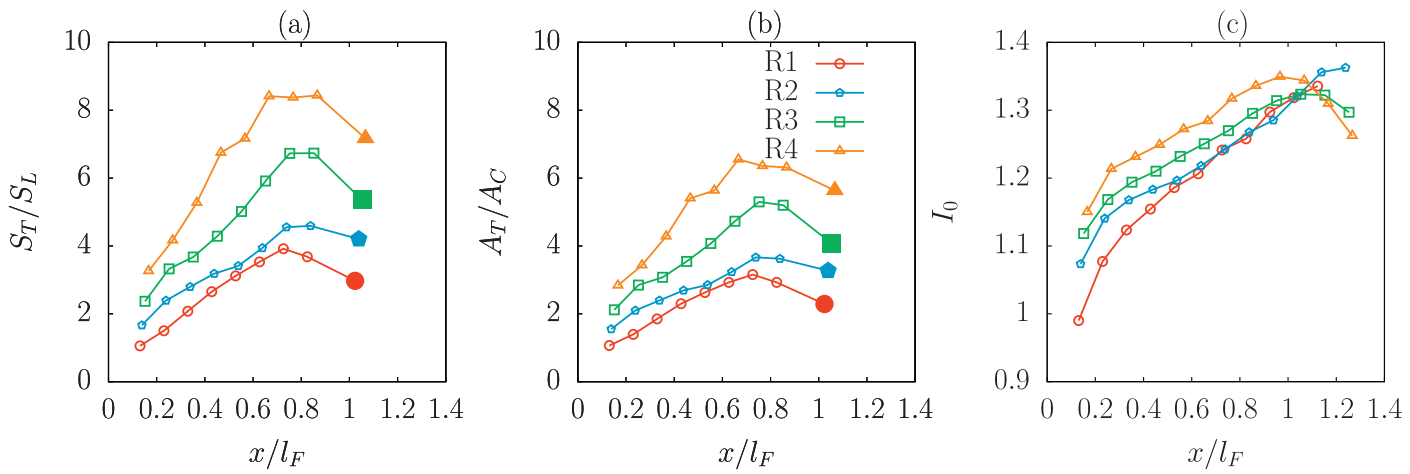

Fig. 3. Evolution of the turbulent flame speed (a), ratio of the turbulent and reference flame area (b), and factor $I_{0}=$ $\left(S_{T} / S_{L}\right) /\left(A_{T} / A_{C}\right)$ (c) in the streamwise position $x$, normalized with the flame length $l_{F}$, for the four flames at different Reynolds numbers: $\mathrm{R} 1 \mathrm{Re}=2800$ (red circles), $\mathrm{R} 2 \mathrm{Re}=5600$ (blue pentagons), $\mathrm{R} 3 \mathrm{Re}=11200$ (green squares), $\mathrm{R} 4 \mathrm{Re}=22440$ (orange triangles). The filled symbol at $x / l_{f}=1$ for each flame corresponds to statistics computed in the large region around the flame tip identified by the box number 2 in Fig. 2. (For interpretation of the references to color in this figure legend, the reader is referred to the web version of this article.)

inlet conditions for the velocity field are obtained from four auxiliary simulations of fully developed turbulent channel flow.

The reactive, unsteady Navier-Stokes equations are solved in the low Mach number limit [11]. All transport properties are computed with a mixtureaverage approach [12] and a skeletal methane mechanism with 16 species and 72 reactions [13] is employed. The resolution is such that $\delta_{L} / \Delta \sim 6$ and $\Delta / \eta<2$ at all times. Cases R1 and R2 were simulated also with double the spatial resolution $(\Delta=$ $10 \mu \mathrm{m})$ without any significant change in the statistics. A discussion of the resolution requirements and an assessment of the quality of the solution is discussed by Luca et al. [5].

A two-dimensional cut of the temperature field is shown in Fig. 2a for flame R4, characterized by the highest Reynolds number. An instantaneous isosurface for the value of the progress variable $c$ corresponding to the peak methane mass fraction consumption rate in a one-dimensional unstreatched laminar flame is shown in Fig. 2b. The progress variable is based on the methane mass fraction $c=1-\left(Y_{\mathrm{CH}_{4}} / Y_{\mathrm{CH}_{4}, \text { in }}\right)$, where $Y_{\mathrm{CH}_{4} \text {, in }}$ is the mass fraction of methane in the unburnt mixture injected in the central jet.

It is evident that the flame surface is characterized by wrinkling over a large range of scales and the overall flame size, which exceeds 400 laminar flame thicknesses in the streamwise direction, is large. Three isocontours of the mean progress variable are also shown to identify the flame brush and its growth in the streamwise direction. After a transitional region close to the jet nozzle, the flame brush grows linearly up to about $80 \%$ of the flame length $l_{F}$, defined as the position on the centerline where the mean reaction rate has the maximum. After this points, a very strong curvature of the isocontour of the mean field is observed and the flame brush becomes about one order of magnitude larger than before. Along its length, the flame can be divided in three main regions: (i) a flame base region where the Kelvin-Helmholtz instability [14] develops into turbulence, (ii) a fully developed turbulent flame characterized by rather small mean curvature, (iii) a flame tip region. While the separation between these three regions is clear for the high Reynolds number case shown in Fig. 2, the flames at lower Reynolds numbers show a progressive reduction in the size of the central, fully developed, turbulent region and for case R1, the transitional region near the base overlaps with the flame tip $[5,15]$.

\section{Definition of the turbulent flame speed}

Due to the strong spatial inhomogeneity of the flow and turbulent flame in the streamwise direction $x$, it is appropriate to define a local turbulent flame speed which evolves in the streamwise direction. In the present study, the turbulent flame speed is defined as a turbulent consumption speed, based on the reaction rate of the methane mass fraction. First, the streamwise direction is divided in a number of volumes $\mathcal{V}(x)$. Each of these extends along the entire spanwise $z$ and crosswise direction $y$. The size of these volumes in the streamwise direction is as small as possible, yet guarantees converged statistics for the area of isosurfaces, which is needed in the analysis. Two examples of (the 2D projection of) these volumes are shown in Fig. 2b by the boxes marked with the numbers 1 and 2 . Fig. 2c,d show enlarged views of the two regions, including a two-dimensional cut of the instantaneous flame surface, defined as the isosurface of the methane-base progress variable field. The value of the progress variable $c=c_{0}=0.73$ is selected as 
it identifies the flame surface corresponding to the peak methane consumption in a one-dimensional unstreatched laminar flame. The area of the flame surface in the volume $\mathcal{V}$ defines an instantaneous flame area $\mathcal{A}$ and its ensemble mean is the average flame surface area $A_{T}$. A two-dimensional projection of a reference area $A_{C}$ is also shown in Fig. 2(c-d); $A_{C}$ is defined from an isosurface of the mean progress variable field using the same value used to define the instantaneous flame surface.

For volume $\mathcal{V}$, the turbulent flame speed is defined as the integral:

$S_{T}(x)=-\frac{\int_{\mathcal{V}} \rho \dot{Y}_{\mathrm{CH}_{4}} d v}{\rho_{u} Y_{\mathrm{CH}_{4}, \text { in }} A_{C}}$

where $\rho$ and $\dot{Y}_{\mathrm{CH}_{4}}$ (in units of time inverse) are the local values of the density and reaction rate of fuel, $\rho_{u}$ is the unburnt gas density, $Y_{\mathrm{CH}_{4} \text {,in }}$ the fuel mass fraction in the unburnt mixture.

While the integral of the reaction rate is defined easily for any volume, the reference area $A_{C}$ requires care. Fig. 2 illustrates the way in which the reference area is defined in the different regions of the flame. For $x / l_{F} \leq 0.8$, the identification of an appropriate reference area for a given volume does not pose issues since the mean flame surface is slightly curved. On the contrary, at the tip, the mean flame surface is oriented in the crosswise direction, so that a volume of limited streamwise size would comprise only a small amount of the instantaneous surface. For this reason, a single volume comprising the entire flame tip region (see region 2 in Fig. 2) is considered and one value of the turbulent flame speed is computed for $x / l_{F} \geq 0.8$.

The turbulent flame speed can be related to the area ratio of the turbulent and mean flame surfaces:

$$
\frac{S_{T}}{S_{L}}=I_{0} \frac{A_{T}}{A_{C}} .
$$

where the coefficient $I_{0}$ accounts for possible deviations from the proportionality between the increase of flame area due to turbulent stirring and the turbulent flame speed. It is worth noting that the computation of the coefficient $I_{0}$ does not require the mean reference area:

$I_{0}=\frac{S_{T}}{S_{L}} \frac{A_{C}}{A_{T}}=\frac{\Omega^{*}}{S_{L}} \frac{1}{A_{T}}$

where

$\Omega^{*}=-\frac{\int_{\mathcal{V}} \rho \dot{Y}_{\mathrm{CH}_{4}} d v}{\rho_{u} Y_{\mathrm{CH}_{4}, \text { in }}}$

is a normalized total volumetric burning rate $\left(\mathrm{m}^{3} / \mathrm{s}\right)$ in the volume $\mathcal{V}$. In addition, the issue mentioned above for an appropriate definition of the turbulent flame speed in the flame tip region does not affect the determination of $I_{0}$ and the definition of $\Omega^{*}$, which may be be computed and analyzed in volumes with a small extent in the streamwise direction in the tip region, also.

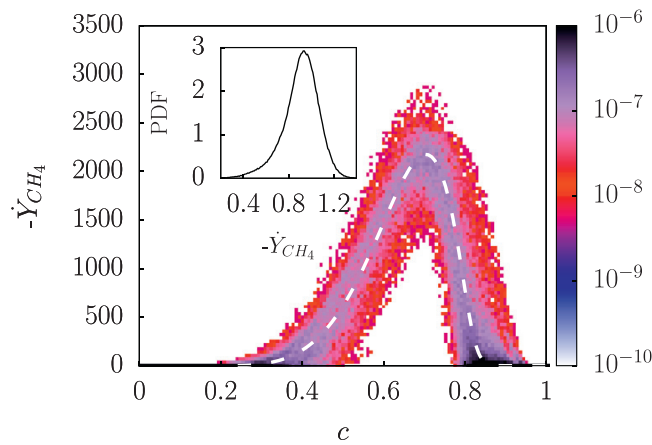

Fig. 4. Joint probability density function of the progress variable $\mathrm{c}$ and the reaction rate of methane in flame R4 characterized by the highest Reynolds number. The white dashed line is the methane reaction rate in a onedimensional laminar flame (flamelet) at the same condition of the turbulent DNS. The inset shows the probability density function of the reaction rate conditioned on the progress variable being $c=0.73$, corresponding to the value where the conditional mean reaction rate peaks. (For interpretation of the references to color in this figure legend, the reader is referred to the web version of this article.)

\section{Results}

The turbulent flame speed computed with Eq. (1) and normalized with the laminar flame speed $S_{L}$ is shown in Fig. 4a for all four flames. The data point at $x / l_{F}=1$ reflects the contribution of the entire flame tip region $x>0.8 l_{F}$ and is marked with a different symbol accordingly. The flame speed increases significantly in the streamwise direction, it reaches a maximum at $x=0.8 l_{F}$ and then decreases slightly to the value at the flame tip. In addition, the flame speed increases monotonically across flames at increasing Reynolds number. Figure $4 \mathrm{~b}$ shows the ratio between the ensembleaveraged turbulent flame area $A_{T}$ and the reference area $A_{C}$. The increase of the burning velocity in the streamwise direction and with Reynolds number correlates well with the increase in the area. Consistently, the final drop of the burning velocity at $x>0.8 l_{F}$ is related to the decrease of flame area. The streamwise evolution of the flame area in these flames has been explained in Ref. [5]; the increase is related to the predominance of the strain term in the total flame stretch, while for $x>0.8 l_{F}$, the propagative-curvature term overcomes strain causing the area to decrease.

It is interesting to note that, while clearly correlated, the turbulent flame speed and the area ratio $A_{T} / A_{C}$ are not proportional. This is more evident in Fig. $4 \mathrm{c}$ where the ratio $I_{0}$ between the turbulent flame speed and the area is plotted. As discussed before, the issue regarding a proper definition of the reference area $A_{C}$ in the flame tip is not relevant here because $I_{0}$ is defined as Eq. (3) 
without computing the reference area. For this reason, the results for $I_{0}$ are available up to $x=1.2 l_{F}$ and with high resolution in the streamwise direction also in the flame tip region. The analysis of Fig. $4 \mathrm{c}$ shows that $I_{0}$ is always larger than one, indicating an enhancement that is not explained by the area ratio $A_{T} / A_{C}$ alone. In addition, the ratio $I_{0}$ increases in the streamwise direction and for increasing Reynolds numbers despite the Karlovitz number remaining nearly constant.

In a previous analysis of the same database performed by Luca et al. [5], it was observed that a global definition of the turbulent flame speed and area indicated a value for the factor $I_{0}$ very close to unity. The global evaluation was based on the assumption of a statistically stationary envelope flame, which implies that (i) the total fuel reaction rate in the entire domain is equal to the flux of fuel from the inlet and (ii) the total mass flux across the flame surface is equal to the flux of the reactants from the inlet. The latter assumption might be inaccurate in high $\mathrm{Ka}$ flames and the global indicator might lead to incorrect interpretation of the physics in certain circumstances, when mixing between the fresh gas (jet) and the fully burnt product (coflow) is present, as shown by Wabel et al. [16].

From the definition of the turbulent flame speed, Eqs. (1) and (4), two phenomena may be responsible for the departure of $I_{0}$ from unity: (i) modifications to the reaction rate $\dot{Y}_{\mathrm{CH}_{4}}$, (ii) thickening of the inner reaction layer of the flame. While these two phenomena may be tightly connected because the reaction rate tends to decrease in regions of high curvature, which correlate with lower gradients and flame thickening, their joint statistics are not trivial and more analysis is required.

Figure 4 shows the joint probability density function (JPDF) of the progress variable $\mathrm{c}$ and methane reaction rate $Y_{\mathrm{CH}_{4}}$. Also shown is a comparison with a flamelet obtained in a onedimensional freely propagating unstretched laminar flame. First, it is observed that the scatter introduced by turbulence is rather limited. The JPDF decreases rapidly moving away from the most probable value at each progress variable. This is highlighted in the inset of the same figure, showing the pdf of the methane reaction rate conditioned on $c=0.73$, where the peak reaction is located. Again, the pdf is rather narrow with probability of deviation of $\pm 20 \%$ from the most probable value being less then $10 \%$ of the maximum probability. The comparison with the laminar flamelet highlights that the highly probable values in the DNS at each progress variable practically matches the flamelet solution. This analysis suggests that the variation of $\dot{Y}_{\mathrm{CH}_{4}}$ is likely to play only a minor role. This is verified in Fig. 5, where the quantity $\Omega^{*}$ (Eq. (4)), is compared with the value obtained computing the integral in Eq. (4) with the flamelet reaction rate (white dashed line in Fig. 4) in place of the actual DNS value $\dot{Y}_{\mathrm{CH}_{4}}$. The comparison shows

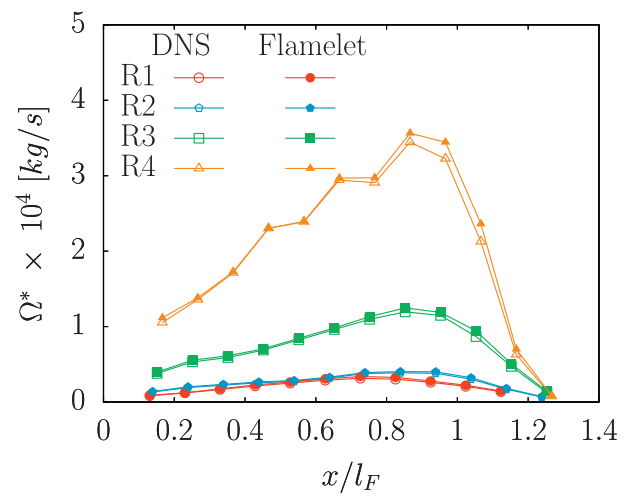

Fig. 5. Comparison of the integrated reaction rate $\Omega^{*}$ (Eq. (4)) obtained from DNS data and using a flamelet model for the local reaction rate.

that the effect of turbulence on $\dot{Y}_{\mathrm{CH}_{4}}$ has a negligible impact on the overall fuel consumption rate and therefore a negligible effect on the turbulent flame speed. The value computed with the flamelet reaction rate is slightly larger than that computed from the actual DNS; therefore, if any, the effect of turbulence on the local reaction rate induces $I_{0}$ to drop below unity, as it is usually observed in very high Karlovitz flames $[17,18]$.

From the discussion above, the value of $I_{0}>1$ must be related to a thickening of the reaction layer of the flame that is not associated with a decrease of the reaction rate. In order to quantify this effect, we consider a region of the flow, e.g., one of the volume used for the turbulent flame speed in Fig. 2, and compute the volume of fluid between the flame surface, identified by $c=c_{0}=0.73$, and a second isosurface $c=c_{k}$; then, the volume of fluid is normalized by the area of the flame surface to obtain a measure $\Psi$ of the distance between the flame and the isosurface $c=c_{k}$. In particular, $\Psi=1 / A_{T} \int_{\mathcal{V}} \mathcal{H}\left(c_{0}-c(\mathbf{x}, t)\right) \mathcal{H}\left(c(\mathbf{x}, t)-c_{k}\right) d v$ for $c_{k}<c_{0}$ and $\Psi=1 / A_{T} \int_{\mathcal{V}} \mathcal{H}\left(c(\mathbf{x}, t)-c_{0}\right) \mathcal{H}\left(c_{k}-\right.$ $c(\mathbf{x}, t)) d v$ for $c_{k}>c_{0}$, where $\mathcal{H}$ is the Heaviside function. Values of $\Psi$ are shown in Fig. 6 for the flames R2 and R4. The same analysis is shown also for a 1D planar laminar flame, taken to represent the baseline in the absence of thicknening induced by turbulence. The figure shows significant thickening for R2 and R4 in the inner layer of the flame $\left(0.5<c_{k}<0.8\right)$, in addition to the welldocumented thickening of the preheat region observed in the range of Karlovitz numbers characteristic of the present flames. Even more interesting is the observation that the thickening increases with Reynolds number. In particular, the inset shows that the ratio between the thickness observed in the $\mathrm{R} 4(\mathrm{Re}=22400)$ and $\mathrm{R} 2(\mathrm{Re}=5600)$ flames is significantly above one.

The visualization of the progress variable field in Fig. 7 shows a region, marked by the black 


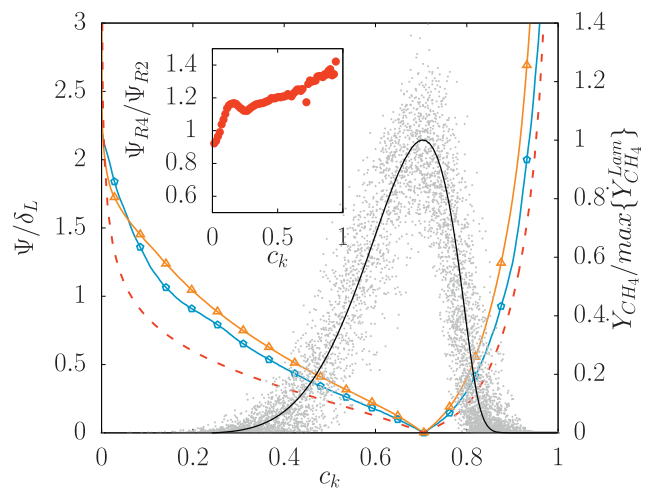

Fig. 6. Volume of the fluid between the flame isosurface $c=0.73$ and isosurface $c_{k}$ as a function of $c_{k}$, normalized by the area of the isosurface $c=0.73$ and the laminar flame thickness. The results for the R2 (blue pentagons) and R4 (orange triangles) flame are compared with those in a laminar flame (red dashed line). The scatter represents the reaction rate of methane and identifies the reaction layer, while the solid black line is the reaction rate in a laminar 1D flame, both normalized with the peak in the laminar flame $\max \left\{\dot{Y}_{\mathrm{CH}_{4}}^{\mathrm{Lam}}\right\}$. The inset shows the ratio between the R4 and R2 cases. Data are from the volume marked as 1 in Fig. 2 and spanning the streamwise locations $0.55<x / l_{F}<0.65$. (For interpretation of the references to color in this figure legend, the reader is referred to the web version of this article.)

box, where a significant thickening of the reaction layer is present. While for clarity the visualization is shown for a two-dimensional cut, it has been verified that the highlighted structures are not thick because of out-of-plane effects. It is worth noting that the thickened region has a size of the order of the integral scale. Other thickened regions are also evident and have a similarly large size. This observation suggests that the thickening events are related to relatively large and intermittent turbulent structures [19] which contain enough energy to modify the inner flame thickness while not affecting the reaction rate significantly. Since the average size of the largest turbulence structure $(l)$ increases with $\mathrm{Re}$ at constant $\mathrm{Ka}$, these regions characterized by thickening get larger and thicker for higher $\mathrm{Re}$, consistently with the increase of the normalized volume $\Psi$ shown in Fig. 6.

All flames in the present study are characterized by similar turbulence intensity $u^{\prime} \approx 10 S_{L}$ and similar Karlovitz number $\mathrm{Ka}=20$, so the only parameter responsible for the Reynolds number variation across simulation is the integral scale of turbulence $l$, which is varied by changing the jet width $H$. A direct analysis of the effect of the integral scale is shown in Fig. 8. The turbulent flame speed $S_{T} / S_{L}$, area ratio $A_{T} / A_{C}$, and the factor $I_{0}$ are plotted versus the local integral scale for all available streamwise locations and flames. The integral scale is defined for each streamwise location at the cross-

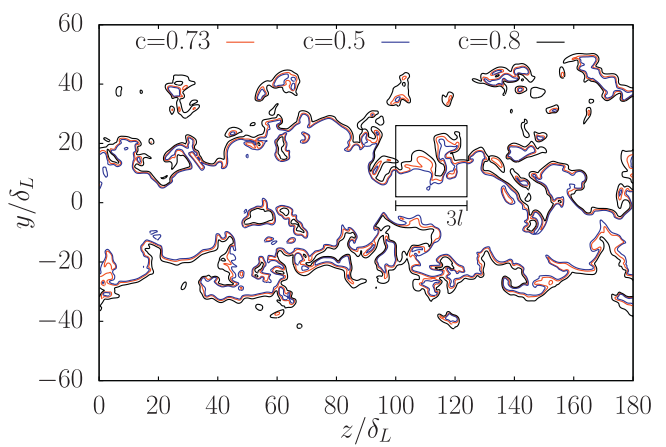

Fig. 7. Visualization of the progress variable field in the $y-z$ plane at $x=0.6 l_{F}$ for the highest Reynolds number flame (R4). The isocontours of the three value of progress variable corresponding to the flame surface, i.e., maximum reaction rate, $(c=0.73$, red $)$ and to about $20 \%$ of the peak reaction rate on each side of the flame $(c=0.5$, blue and $c=0.8$, black) are plotted. The box marks a region, of size $3 l$, where significant thickening of the reaction layer is observed. (For interpretation of the references to color in this figure legend, the reader is referred to the web version of this article.)

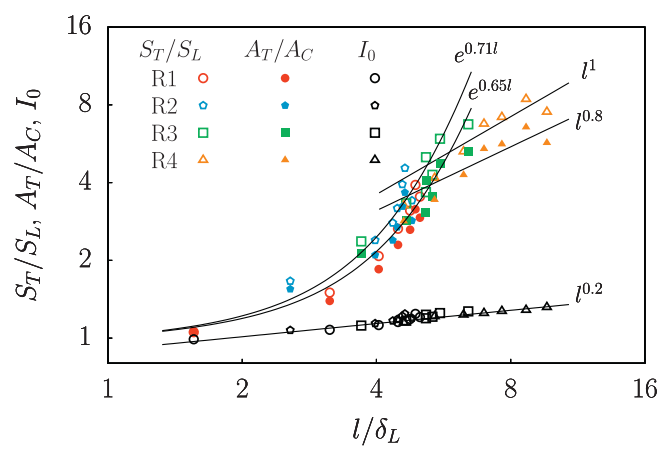

Fig. 8. Turbulent flame speed (color open symbols), ratio of the turbulent and reference flame area (color filled symbols), and factor $I_{0}=\left(S_{T} / S_{L}\right) /\left(A_{T} / A_{C}\right)$ (black open symbols) versus the integral scale of turbulence $l$ for flames at different Reynolds numbers: $\mathrm{R} 1 \mathrm{Re}=2800$ (red circles), $\mathrm{R} 2 \mathrm{Re}=5600$ (pentagons), R3 Re $=11200$ (squares), $\mathrm{R} 4 \mathrm{Re}=22440$ (triangles). The lines are exponential and power-law fits of the form indicated in the figure. (For interpretation of the references to color in this figure legend, the reader is referred to the web version of this article.)

wise position where $\langle c\rangle=0.73$. As expected, the flame speed and area ratio grow with the integral scale [6-8]. In an attempt to assess a scaling law, several curve fits for the flame speed and area ratio with respect to $l$ are included in the figure. It is observed that for relatively small integral scales, up to a value of $l$ corresponding to about 6 laminar flame thicknesses, the flame speed and area ratio increases exponentially. For larger values of $l$, a power-law fitting is more appropriate with a 
scaling of $l^{1}$ and $l^{0.8}$ for the flame speed and area ratio, respectively. Given the power-law scaling $l^{1}$ and $l^{0.8}$ for large $l$, the factor $I_{0}$ should scale as $l^{0.2}$. This scaling is also reported in the figure. While some uncertainty in the scaling exponents of the powerlaw for the flame speed, area ratio, and $I_{0}$ is evident, the observation that the increase of $I_{0}$ with the integral scale, or equivalently, that the inner flame layer becomes thicker for larger values of the integral scale, appear to be conclusive. The same scaling is observed also if the turbulent flame speed, ratio of the turbulent and reference flame area, and the factor $I_{0}$ are plotted versus the turbulent Reynolds number $\operatorname{Re}_{\mathrm{T}}=u^{\prime} l / v$; the plot and a brief discussion are reported in the supplementary material. This last observation confirms that the minor variations of Karlovitz number across the flames do not play an important role.

The role of the integral scale $l$ at constant Karlovitz number has been recently investigated by Lapointe [7] in homogeneous isotropic turbulence; in that work, the factor $I_{0}$ and inner flame thickness were found to be independent of $l$. There are several circumstances that might explain the difference. First, the presence of shear in the present jet configuration might play a role. However, shear decreases with the Reynolds number and downstream distance in the present configuration and, usually, it tends to have a decreasing impact on the dynamics of turbulence as the Reynolds number increases [14,20-22], likely implying that shear might not be the reason of the observed behavior. Moreover, the flames considered by Lapointe [7] are characterized by a rather small ratio between the inner flame layer and the thermal thicknesses; in the present work, due to the high temperature of the fresh mixture, the reaction layer is rather thick and therefore more prone to be affected by turbulence. It is then possible that the values of integral scale considered by Lapointe, ranging between 1 and 4 flame thicknesses, are too small to highlight the effects on $I_{0}$. Finally, Fig. 7 shows that the regions where the thickening of the inner layer is observed are rather large, of the order of 3 integral scales. Likely, this fact implies that a large domain is required to avoid boundary effects. Therefore, the limited domain size employed in the work of Lapointe [7], which is fixed at 5 integral scales due to the linear forcing employed, might limit the effect of the integral scale on $I_{0}$. Nevertheless, the data currently available do not allow a systematic assessment of all the possible reasons of the difference and additional analyses will certainly be of interest.

The conclusion that the factor $I_{0}$ and the thickening of the inner layer increase with Reynolds number at nearly constant Karlovitz is a significant result. It points to the possibility that, contrary to the classical theory describing the effects of the two main parameters of the Borghi-Peters diagram ( $l$ and $u^{\prime}$ or Re and $\mathrm{Ka}$ ), an increase of $l$ or $R e$ at constant $u^{\prime}$ or Ka might cause variations in $I_{0}$ and thickening of the inner layer, which are usually ascribed solely to variations in $u^{\prime}$ and $\mathrm{Ka}$. This result is, to a certain extent, consistent with the recent work of Driscoll et al. [4] who reported a new measured diagram of combustion in which a regime characterized by a broadened preheat layer appears increasing the integral scale $l$ at constant turbulent intensity $u^{\prime}$.

Based on the data and discussions above, we speculate that the transition from the thin reaction zone regime to distributed burning [23], in which turbulence alters transport in the inner layer, might occur at lower values of Ka for high Re (large integral scales) compared to the value of Ka needed to observe distributed burning at low Re (small integral scale). In other words, the transition may depend on both Ka and Re. Direct verification of this conjecture would require additional simulations at different values of Ka for each Re considered in the present flame set.

\section{Conclusions}

The data from four DNS of turbulent premixed slot jet flames up to $\mathrm{Re}=22000$ show that the turbulent flame speed, evaluated at each streamwise position, increases with the distance from the nozzle and with the Reynolds number of the flame. This is not entirely due to the increase of the flame area since a significant thickening of the inner reaction layer, associated with negligible changes of the reaction rates, also contributes to the enhancement of the turbulent flame speed. It is concluded that, even if the flame are characterized by a constant Karlovitz number, an higher Reynolds number, and correspondingly larger turbulence integral scale, enhances the effect of turbulence inside the inner flame layer.

\section{Declaration of Competing Interest}

None.

\section{Acknowledgements}

The authors acknowledge funding from of the European Research Council (ERC) under the European Unions Horizon 2020 research and innovation program under grant agreement no. 695747.

\section{Supplementary material}

Supplementary material associated with this article can be found, in the online version, at doi: 10. 1016/j.proci.2020.06.210. 


\section{References}

[1] N. Peters, Turbulent Combustion, Cambridge university press, 2000 .

[2] D. Veynante, L. Vervisch, Prog. Energy Combust. Sci. 28 (3) (2002) 193-266.

[3] J.F. Driscoll, Prog. Energy Combust. Sci. 34 (1) (2008) 91-134.

[4] J.F. Driscoll, J.H. Chen, A.W. Skiba, C.D. Carter, E.R. Hawkes, H. Wang, Prog. Energy Combust. Sci. 76 (2020) 100802.

[5] S. Luca, A. Attili, E. Lo Schiavo, F. Creta, F. Bisetti, Proc. Combust. Inst. 37 (2) (2019) 2451-2459.

[6] N. Peters, J. Fluid Mech. 384 (1999) 107-132.

[7] S. Lapointe, Simulation of Premixed Hydrocarbon Flames at High Turbulence Intensities, Ph.D. thesis, California Institute of Technology, 2016.

[8] T. Kulkarni, A. Attili, F. Bisetti, Reynolds number scaling of burning rates in spherical turbulent premixed flames, J. Fluid Mech. (under review).

[9] A.W. Skiba, T.M. Wabel, C.D. Carter, S.D. Hammack, J.E. Temme, J.F. Driscoll, Combust. Flame 189 (2018) 407-432.

[10] E.R. Hawkes, O. Chatakonda, H. Kolla, A.R. Kerstein, J.H. Chen, Combust. Flame 159 (8) (2012) 2690-2703.

[11] A. Tomboulides, J. Lee, S. Orszag, J. Sci. Comput. 12 (2) (1997) 139-167.
[12] A. Attili, F. Bisetti, M.E. Mueller, H. Pitsch, Combust. Flame 166 (2016) 192-202.

[13] S. Luca, A.N. Al-Khateeb, A. Attili, F. Bisetti, J. Propul. Power (2017) 1-8.

[14] A. Attili, F. Bisetti, Phys. Rev. E 88 (3) (2013) 033013.

[15] S. Luca, A. Attili, F. Bisetti, Direct numerical simulation of turbulent lean methane-air bunsen flames with mixture inhomogeneities, 54th AIAA Aerospace Sciences Meeting, AIAA SciTech Forum, AIAA 2016-0189.

[16] T.M. Wabel, R.S. Barlow, A.M. Steinberg, Combust. Flame 208 (2019) 327-329.

[17] H. Wang, E.R. Hawkes, B. Zhou, J.H. Chen, Z. Li, M. Aldén, Proc. Combust. Inst. 36 (2) (2017) 2045-2053.

[18] H. Wang, E.R. Hawkes, J.H. Chen, B. Zhou, Z. Li, M. Aldén, J. Fluid Mech. (2017) 511-536.

[19] D. Fiscaletti, A. Attili, F. Bisetti, G.E. Elsinga, J. Fluid Mech. 791 (2016) 154-173.

[20] C.M. Casciola, P. Gualtieri, B. Jacob, R. Piva, Phys. Rev. Lett. 95 (2) (2005) 024503.

[21] A. Celani, M. Cencini, M. Vergassola, E. Villermaux, D. Vincenzi, J. Fluid Mech. 523 (1) (2005) 99-108.

[22] A. Attili, F. Bisetti, Phys. Fluids 24 (2012) 035109.

[23] A.J. Aspden, M.S. Day, J.B. Bell, J. Fluid Mech. 871 (2019) 1-21. 